\title{
Sinnvolle Alterität?
}

\author{
Zu unterschiedlichen formalen Entwicklungen \\ in der mittelhochdeutschen, okzitanischen \\ und altfranzösischen Literatur
}

\section{Victor Millet}

\begin{abstract}
This paper analyses the formal structure of Troubadour-and Trouvère poetry, with its great variety of stanza forms and possible stanza combinations, in comparison to German Minnesang, which stays true to a basically monostrophic model based on only one kind of stan$z a$, the Stollenstrophe. The paper suggests that this is not due to lack of capability, but that it must be understood as a conscious characteristic of poets composing songs in a >German< manner. The paper then moves on to strophic narrative poetry as a characteristic of German epics, arguing that in a similar way this development seems to happen in conscious opposition to French narrative, which develops huge prose romances and even in the chanson de geste tends to work with lines as opposed to stanzas or groups of lines.
\end{abstract}

Title: Significant Difference? On Diverging Formal Developments in Middle High German, Occitanic and Old French Literature

Title: comparative literature; medieval literature; metrics; stanza

Die historischen Beziehungen zwischen Frankreich, Okzitanien und Deutschland waren im 12. und 13. Jahrhundert sehr eng und sind uns aus vielen unterschiedlichen Quellen bekannt. Verwandtschaftliche Verflechtungen zwischen den Fürstenhäusern, regelmäßige freundschaftliche und diplomatische Besuche, gemeinsame wirtschaftliche und militärische Interessen, Hoffeste zu unterschiedlichen Anlässen - der mitteleuropäische Adel ist eine schmale Elite, in der man sich über die Grenzen hinweg gut kennt oder viel voneinander gehört hat (vgl. Bumke 1986). Leser, Auftraggeber und Autoren, jedenfalls die in Deutschland, wussten - davon können wir ausgehen - sehr genau, was sich an den Höfen und Literaturzentren jenseits des Rheins tat, denn sie hatten entweder selbst die Gelegenheit gehabt, es zu hören, oder sie hatten davon berichten gehört. Dennoch, und auch trotz der in jener Zeit noch alles umfassenden latinitas im Sinne einer lateinischen Bildung und Kulturtradition, weisen die literarischen Traditionen in den Volkssprachen charakteristische Unterschiede auf, die nicht immer leicht zu erklären sind. Ich will in diesem Beitrag versuchen, die Ungleichheiten in den literarischen Formen zu analysieren, sowohl in der Lyrik wie in der Epik. Selbstverständlich geben sie allein nicht Aufschluss über die Unter- 
schiede in der literarischen Entwicklung diesseits und jenseits der Sprachgrenze, aber sie können helfen zu erklären, warum und vor allem mit welchen Mitteln man eigene Interessen verfolgt hat.

Die mittelhochdeutsche Lieddichtung beginnt, wenn unsere Datierungen richtig sind, mit einigen mit der provenzalischen und französischen Produktion nur schwach in Verbindung zu bringenden Vorläufern, dem sogenannten donauländischen Minnesang, an dessen Spitze der Kürenberger steht. Soweit wir das aus dem überlieferten Material erkennen können, erhält dieser neue höfische Liedtypus Aufschwung und Konsistenz als Gattung in dem Moment, in dem er sich dezidiert an die okzitanische Troubadour- und an die französische Trouvère-Dichtung anlehnt (vgl. Räkel 1986; Schweikle 1995; Herchert 2010). Die frühe Lyrik weist eine Tendenz zur Einstrophigkeit auf; Lieder mit mehreren Strophen kommen, wenn unsere Datierungen stimmen, erst mit besagter Anlehnung an romanische Vorbilder auf. Doch die Strophik, die der deutsche Minnesang entwickelt, ist in vielen wichtigen Aspekten ganz anders als die der provenzalisch-französischen Vorbilder. Und meine These lautet, dass dieser Unterschied signifikant oder zumindest charakteristisch ist.

In den frühen Liedern finden sich meist recht einfache Strophenformen. Der Kürenberger ist uns vor allem durch seine vierzeiligen, paarweise gereimten Langzeilenstrophen (Ton II, sogenannte Nibelungenlied-Strophe) bekannt. Zweifach paargereimte Kurzverse finden sich bei Dietmar von Aist (z.B. MF 39,18: 4a' 4a' 4b 5b), daneben auch Strophen mit mehreren Reimpaaren (z.B. Dietmar, MF 37,4: sieben Reimpaare). Eine Variante sind die Kombinationen von Lang- und Kurzversen, entweder in beliebiger Zusammenstellung (z.B. Dietmar, MF 32,13: ein Langzeilen-Reimpaar + zwei Kurzzeilen-Reimpaare) oder als Einschub zwischen den Reimpaaren (die sogenannte Stegstrophe, z.B. Kürenberger, MF 71,1: 6a 6a 3b 6c 6c). Doch schon mit Heinrich von Veldeke und Rudolph von Fenis werden Paarreime selten. An ihre Stelle treten sogenannte Periodenstrophen, die Versgruppen (Perioden) mit etwas komplexeren Reimkombinationen enthalten, also Kreuzreime oder Umarmungsreime und ihre Varianten (z.B. Veldeke, MF 66,1: abba cdcd; oder Fenis, MF 84,10: abba cddcc). Doch schon bald darauf erscheint die Stollenstrophe mit ihrer Zweiteilung in Aufgesang und Abgesang, wobei der Aufgesang aus zwei metrisch und musikalisch gleichgebauten Teilen, den sogenannten Stollen, besteht, während der Abgesang von diesem ersten Block in der Regel unabhängig ist, stets frei kombinierbar bleibt und beliebig ausgedehnt werden kann. Dieses im Grunde sehr einfache, abstrakte Formmodell bietet den Vorteil, vielfach ausgestaltet werden zu können: Die Verslänge kann beliebig variiert werden (einschließlich Reimkombinationen von Kurz- und Langversen), die Stollen können auf drei oder vier Verse erweitert und ihre Reimfolge gespiegelt oder kombiniert werden (z.B. Terzinenstollen, Dietmar, MF 40,19: aab ccb); der Abgesang kann ohnehin beliebig abgewandelt, erweitert oder gekürzt werden. Es handelt sich also um ein vergleichsweise einfaches Grundmuster, das zugleich einen breiten Variationsspielraum offenlässt.

1 | Moser/Tervooren 1998 
Seit Einführung der Stollenstrophe operiert der gesamte deutsche Minnesang vom Ende des 12. bis in das 14. Jahrhundert hinein fast ausschließlich mit diesem Modell. Ausnahmen bestätigen die Regel; kein deutscher Dichter hat ernsthaft versucht, andere Liedmodelle durchzusetzen. Die Stollenstrophe steht für ein Kompositionsmodell, in dem die einzelne Strophe im Mittelpunkt steht, denn jede ist in formaler Hinsicht unabhängig, bildet eine Einheit und steht in keinem ordnenden oder hierarchisierenden Zusammenhang zu den übrigen Strophen. Ein deutsches Minnelied ist eine Reihung von Strophen, die zwar dem gleichen metrischen Schema folgen, aber sowohl metrisch wie auch in Bezug auf ihre Reimkombinationen unabhängig voneinander sind. Dies hat sicherlich dazu beigetragen, dass in der deutschen Lieddichtung die Strophenanzahl und die Strophenfolge der Lieder in den verschiedenen Handschriften immer wieder abweichen. Wahrscheinlich sind manche Lieder auch in unterschiedlicher Strophenzahl und -reihenfolge vorgetragen worden.

Es ist das gleiche Prinzip, das auch für die Sangspruchdichtung gilt, nur haben wir es hier mit komplexeren, meist längeren Strophenformen zu tun, die außerdem auch inhaltlich isolierte Einheiten darstellen und damit je neue Kompositionen sind. Sangspruchstrophen im gleichen Ton, also mit der gleichen Strophenform, sind in der Regel zu unterschiedlichen Zeitpunkten entstanden, bilden jedoch Reihen auf der Basis einer weitgefassten inhaltlichen Zusammengehörigkeit. Umso mehr können hier Anzahl und Reihenfolge der Strophen in der handschriftlichen Überlieferung variieren, ja wahrscheinlich sind viele Sprüche erst bei der Notation zusammengekommen - dass dies auch für den Vortrag galt, der wohl meist partiell war, darf als sicher gelten (vgl. Tervooren 1995). Da im Minnesang die außerliterarischen Anspielungen auf Personen oder Begebenheiten fehlen, die uns in der Sangspruchdichtung oft genug erlauben, von unterschiedlichen Kompositionsmomenten $\mathrm{zu}$ sprechen, können wir für die Liebeslyrik nicht bestimmen, ob auch hier eine zeitlich abgesetzte Komposition der Strophen gewöhnlich war. Wir wissen schlicht zu wenig von den möglichen Kompositionsprozessen mittelalterlicher Lieder.

Wer das System des Minnesangs mit dem seiner romanischen Vorbilder vergleicht, dem stellt sich alsbald die Frage, warum die deutschen Dichter, die doch im Übrigen so viel von Provenzalen und Franzosen genommen haben, im formalen Bereich gänzlich unabhängig geblieben sind. Denn in der Romania gibt es keine Stollenstrophen.

Die Strophe der Troubadour-Lyrik kennt die Zweiteilung (bzw. Dreiteilung) der Stollenstrophe nicht. Strophen sind in sich geschlossene, nicht teilbare Konstruktionen. Im Umfang der Strophe sind die Unterschiede gering: Am häufigsten sind in der Romania die Strophen zu acht Versen, häufig sind ebenfalls solche zu neun oder auch zehn Versen; das wird auch die mehrheitliche Länge der deutschen Lieder sein. Aber die romanischen Strophen sind vor allem in Bezug auf die Reimkonstruktion wesentlich komplexer. ${ }^{2}$ Das Modell, das wir aus dem

2 | Vgl. allgemein Asperti 2002, Hasenohr 1990 u. Meneghetti 1984. Zum Folgenden: Di Girolamo 1979; Mölk 1982; Rieger 1983; Riquer 1983. 
Minnesang kennen, nämlich ein in allen Strophen gleiches Reimschema, doch mit von Strophe zu Strophe wechselnden Reimen und innerhalb der Strophe jeweils drei oder vier verschiedene Reime, ist in der Romania eigentlich nicht bekannt. Am ähnlichsten sind dem deutschen Typus die provenzalischen coblas singulars, was so viel bedeutet wie alleinstehende Strophen oder aber auch einheitliche Strophen. In ihnen haben die Strophen gleiche Reimstruktur, doch die Reime sind in jeder von ihnen anders. Die reinste Form dieses Strophenmusters (z.B. Raimbaut de Vaqueiras, Altas undas que venez suz la mar, P/C 392,5a, I: aaaa, II: bbbb, III: cccc, usw.) ist selten; sie findet in Deutschland allerdings eine Entsprechung in Walthers Vokalspiel ( $\mathrm{L}^{4} 75,25$ Diu welt was gelf, rôt und blâ). Das zeigt, dass die romanischen Formen in Deutschland bekannt waren und nachgebildet werden konnten. In der Regel aber haben die coblas singulars noch weitere Reime (z.B. Raimbaut de Vaqueiras, Kalenda maia, P/C 392,9, I: aabaaabababaaacaaaca, II: ddedddedededddfdddfd). Und meistens bleibt einer dieser Reime über alle Strophen hinweg unwandelbar (z.B. Guilhem de Peitieu, Farai un vers de dreit nien, P/C 183,7, I: aaabab, II: cccbcb, III: dddbdb, usw.). Am Beispiel dieses Strophentypus zeigt sich, wie die Troubadour-Dichtung die Strophen formal enger miteinander verknüpft, indem ein festes Element in ihnen wiederholt wird (vgl. Billy 1989). Die Strophen sind nicht alleinstehende Einheiten, wie im Minnesang, sondern sie bilden mit den anderen Strophen ein mehr oder weniger stilisiertes Konstrukt. Hierzu stellt sich auch das poetische Mittel des mot-refranh, ein Refrain-Wort, das sich an gleicher Position in allen Strophen wiederholt (z.B. Raimbaut de Vaqueiras, Gaita be, gaiteta del chastel, P/C 392,16a, I: ababacabbc, wobei der c-Reim ein mot-refranh, also stets das gleiche Wort ist). Auch dieses Element kennt der Minnesang nur in einer sehr einfachen Variante, z.B. in Heinrichs von Morungen Tagelied (MF 143,22), in dem jede Strophe mit demselben Refrain-Wort ansetzt. ${ }^{5}$ Aber es hat sich in der deutschen Lieddichtung nicht verbreitet.

Am häufigsten sind in der provenzalischen Dichtung jedoch nicht die coblas singulars (diese sind deutlich in der Unterzahl), sondern die coblas unissonans (oder eintönige Strophen), in denen die Reime sich in jeder Strophe wiederholen (z.B. Bernat de Ventadorn, Can vei la lauzet mover, P/C 70,43, I: ababcdcd, II: ababcdcd, usw.). Dabei ist es beliebig, wie die Strophe genau konstruiert ist. In jedem Fall erfordert sie ein beachtliches Repertoire an Reimwörtern. Die radikale Form dieses Modells ist die, in der jede Reimposition denselben Reim führt (z.B. Guilhem de Peitieu, Companho, farai un vers qu'er covinen, P/C 183,3, I: aaa, II: aaa, III: aaa, usw.). Eine weitere, hoch kunsthafte Abwandlung dieses

3 | Pillet/Carstens 1933.

4 | Bein 2013.

5 | Onomatopoetische Einlässe wie tandaradei (Walther von der Vogelweider, Under der linden, L 39,11) oder traranuretun traranuriruntundeie (Neidhart, Sommerlied 1 , W/F [Müller u.a. 2007] 3,1) würde ich nicht als Entsprechung zum mot-refranh sehen, weil ein mot ja ein Wort ist, sondern als musikalische Refrains, die lautlich begleitet werden, aber semantisch leer sind. 
Reimmusters sind die coblas unissonans dissolutas (aufgelöste Strophen), in denen die Verse der ersten Strophe alle jeweils unterschiedliche Reime führen, die ihre Entsprechung erst in der zweiten und in den folgenden Strophen finden (z.B. Marcabru, Contra l'iverns que s'enansa, P/C 293,14, I: abcdef, II: abcdef, III: abcdef, usw.). Eine weitere, sehr häufige Variante der unissonans stellen die coblas doblas oder gedoppelte Strophen dar, in denen die Reime in je zwei Strophen identisch sind (z.B. Bernat de Ventardorn, Lo gens temps de pascor, P/C 70,28, I: aaaaaaaa, II: aaaaaaaa, III: bbbbbbbb, IV: bbbbbbbb, usw.); Varianten dieses Typus sind die coblas ternas, in denen gleich drei Strophen identische Reime haben, die coblas quaternas (vier Strophen) oder die coblas alternadas, in denen die ungeraden Strophen stets die gleichen Reime tragen und die geraden Strophen ebenfalls (z.B. Bernat de Ventadorn, Non es meravelha s<eu chan, P/C 70,31, I: abbacddc, II: cbbcadda, III: abbacddc, IV: cbbcadda, usw.). Eine hoch künstlerische Variante dieses alternierenden Modells sind die coblas retrogradadas oder gespiegelte Strophen, in denen sich die Reime in allen Strophen wiederholen, aber in je zwei Strophen in gespiegelter Reihenfolge auftreten (Bsp. 1: Grimoart, Lanquan lo temps renovelha, P/C 190,1, I: abcdefgh, II: hgfedcba, III: abcdefgh, IV: hgfedcba, usw.; Bsp. 2: Guilhem Peire de Cazals, Eras, pus vey mon benastruc, P/C 227,3, I: abccba, II: abccba, usw.). Diese hoch komplexen Formen wurden oft kombiniert mit einer anderen Variante, den coblas capcaudadas oder Kopf-anKopf-Strophen, in denen der Reim des letzten Verses der ersten Strophe im ersten Vers der zweiten Strophe wiederholt wird (Bsp. 1: Bernat de Ventadorn, Tant ai mo cor ple de joya, P/C 70,44, I: ababababcccb, II: bdbdbdbdcccd, III: dedededeccce, usw.; Bsp. 2: Guillem de Cabestany, Ar vey qu'em vengut als jorns loncs, P/C 213,3, I: aababbc, II: ccdcdde, III: eefeffg, usw.). Diese Formmodelle, die die Reihenfolge der Strophen im Lied sowohl für den Vortrag wie auch für die schriftliche Überlieferung fixieren, sind kombinierbar mit noch einem weiteren Mittel, dem der sogenannten coblas capfinidas, in denen das Reimwort des letzten Verses der ersten Strophe zu Beginn des ersten Verses der zweiten Strophe wiederaufgenommen wird (z.B. Arnaut Daniel, Chanson so -ill mot son plan e prim, P/C 29,6, I: ... dels auzels per la bruoilla. II: Pel bruoill aug lo chan e.l refrim ...... on plus vas mi s'orguoilla. III: Val orguoill petit d'amador ... ... qi'incontra'amor ianguoilla. IV: Per ianguoill ges no $m$ vir aillor ... usw.).

Und viele dieser strophischen Konstruktionsformen lassen sich miteinander kombinieren, z.B. singulars und capcaudades oder unissonans und capcaudadas. Die gleichen Kombinationen sind auch mit retrogradadas möglich oder mit capfinidas. Hinzu kommen außerstrophische Elemente wie der refranh (der Refrain), der teilweise in die Strophe eingebettet sein kann, die tornada (kürzere, meist am Ende stehende Versgruppen außerhalb des Strophenverbunds) oder der bereits genannte mot-refranh. Das Repertoire scheint schier unendlich und hat bereits früh zur Katalogisierung der reim-metrischen Muster geführt (vgl. Frank 1966). Aus dieser Perspektive wird verständlich, warum sich in der Romania Liedtypen herauskristallisieren, die nicht durch ihren Inhalt, sondern durch ihre Formkonstruktion bestimmt werden. Eines der verbeitetsten ist sicherlich die dansa; sie besteht aus einem gewöhnlich vierzeiligen Refrain (bekannt als res- 
pós) und Strophen (gewöhnlich sechs- bis achtzeilig), deren vier letzte Zeilen sich auf diesen Refrain reimen (z.B. Guiraut d'Espanha, Domna, sitot no·us es preza, P/C 244,1, R: ABAB, I: cdcdabab, II: efefabab, usw.). Mit ihr verwandt, wenn auch seltener, ist die balada; sie ist dadurch gekennzeichnet, dass sie den Refrain nicht nur als separaten Liedteil hat, sondern ihn gleichzeitig in die Strophen einbaut (Bsp. 1: anonym, D'amor m'estera ben e gent, P/C 461,73, R: AA, I: bAbaAA; Bsp. 2: Cerverí de Girona, Si voletz que $\cdot$ m laix d'amar, P/C 434a,65, R: ABAB, I: aAbBababABAB, II: bBaAbabaABAB). Ebenso verwandt mit diesen beiden ist die viadeira, die vom zweizeiligen refranh nur den zweiten Vers am Ende der Strophen wiederholt, den ersten aber durch ein Spiel variierender Wiederholungen am Anfang der dreizeiligen Strophen abwandelt (z.B. Cerverí de Girona, No.l prenatz lo fals marit, P/C 434a,34, R: AB, I: ccB, II: aaB, III: ccB, IV: aaB, usw.). Formal differenzierte Liedtypen sind zudem die estampida (Strophen mit kontrapunktischen Responsionen, z.B. Raimbaut de Vaqueiras, Kalenda maia, P/C 392,9, s.o.), die retroencha (ähnlicher Aufbau, jedoch mit refranh) oder der descort (ein metrisch und reimmäßig völlig ungeordnetes Lied).

Dieser formale Anspruch der Troubadour-Dichtung, der einen großen Reichtum an poetischen Mitteln entfaltet und zu virtuosen Konstruktionen führt, hat im deutschen Minnesang keine Entsprechung gefunden. Es gibt zwar hier und dort ein paar Ausnahmen, doch sie bleiben isolierte Lieder. Insgesamt gesehen ist der Minnesang, im Vergleich zur romanischen Lieddichtung, eine - provozierend formuliert - formal unterkomplexe Kunstgattung. Die Sangspruchdichtung erreicht strophenintern zwar deutlich größere Kunstfertigkeit, doch zwischenstrophische formale Verknüpfung sind naturgemäß inexistent, weil die Strophen ja Einzeldichtungen sind.

Man wird nicht behaupten wollen, die deutschen Lieddichter hätten diese formalen Finessen ihrer romanischen Zeitgenossen nicht gekannt. Wir wissen von einem intensiven Austausch, von thematischen Anlehnungen und sogar von Kontrafakta, die von genauer Kenntnis der okzitanischen und französischen Dichtung zeugen (vgl. Zotz 2005). Wir haben es zudem mit einer kulturellen Elite zu tun, mit einer schmalen Schicht von Kennern, die leicht die Besonderheiten erkennen und unter denen die Information rasch vermittelt ist. Gleichzeitig wird man nicht annehmen wollen, dass die deutschen Dichter technisch nicht in der Lage gewesen wären, solche Reimkonstruktionen herzustellen, denn es gibt genügend Texte, die das Gegenteil belegen; auch nicht, dass die deutsche Sprache oder Metrik weniger für sie geeignet sei.

Die einzige Erklärung für diese formale Alterität des Minnesangs im Vergleich zur Troubadour- und Trouvère-Lyrik ist, dass sie gewollt ist, dass sich die deutschen Dichter bewusst dafür entschieden haben, formal einen anderen Weg $\mathrm{zu}$ gehen, als ihre provenzalischen und französischen Kollegen. Und sie haben sich offensichtlich im Laufe der Jahre und Jahrzehnte immer wieder dazu entschlossen, d.h., die Wahl ist kontinuierlich beibehalten worden, von jedem Dichter, von jedem Auftraggeber und in jedem Lied. Alle deutschen Dichter haben sich auf formaler Ebene der von ihren Vorgängern gewählten Option angeschlossen. Ein deutsches Lied zu dichten bedeutete offenbar so viel, wie es nach 
dem Modell isolierter Stollenstrophen zu komponieren. ${ }^{6}$ Alles andere wäre wohl als ein Lied in >welscher < Manier verstanden worden. Inwieweit sich dies auch in der Musik niederschlug, können wir nicht wissen, weil die Vergleichsbasis der Melodien zu gering ist. Doch die formale Konstruktion der Texte weist auf eine bewusste und damit sinntragende Opposition der deutschen Dichter gegenüber ihren romanischen Kollegen. Und sie nimmt in Kauf, dass dadurch die Strophen umstellbar waren, ja möglicherweise hat das deutsche Publikum in dieser Umstellbarkeit einen besonderen Reiz erkannt.

Hierzu addiert sich auch die größere Abstraktheit der deutschen Lieder, das Fehlen von Namen, Orten, Zeitgeschehen, die Bedeutung der Gedankenführung und der Innenperspektive, kurz: alles, was Nicola Zotz 2005 unter dem Begriff der >Offenheit < zu fassen sucht; und natürlich auch die unterschiedliche Metrik, an der die deutschsprachigen Autoren ebenfalls festhalten.

Aus dieser Perspektive betrachtet, gerät ein anderes Phänomen romanischdeutscher Unterschiede in der literarischen Formenwahl in ein neues Licht: das Erzählen im Paarreimvers und in Strophen. Der deutsche erzählende Paarreimvers ist nicht aus Frankreich übernommen worden, jedenfalls gab es ihn schon lange, bevor die höfische Erzählliteratur Frankreichs in Deutschland rezipiert und verarbeitet wurde. ${ }^{7}$ Aber die Übereinstimmung des paargereimten Vierhebers mit dem durch Wace, Marie de France und Chrétien de Troyes verbreiteten, paargereimten Achtsilber vereinfachte sicherlich die Übernahme der neuen höfischen Romane. Doch als dann um die Wende zum 13. Jahrhundert in Frankreich, vor allem im Kontext der Fortsetzungen des Gralsromans, die Diskussion um Wahrheit und Lüge aufkam und sich ein Wechsel vom Vers, der als Form des Fiktionalen gebrandmarkt wird, hin zur neuen volkssprachlichen Prosa vollzieht, die dank der lateinischen Prosachronistik den Anspruch der Wahrheit erheben durfte (vgl. Cirlot 1987) und die spätestens mit dem Prosa-Lancelot konsolidiert war, da hat das deutsche Literaturpublikum diesen Wechsel nicht mitmachen wollen und hat bis in das späte 15. Jahrhundert weiterhin in Paarreimversen gedichtet. Während also in Frankreich die Prosa den Roman beherrscht und der Paarreimvers ab dem zweiten Viertel des 13. Jahrhunderts mit einigen Ausnahmen nur noch für die kleineren Erzählformen, also für Fabeln und Fabliaux und ähnliche Textsorten, verwendet wird, bleibt man im deutschsprachigen Bereich beharrlich beim Paarreimer. Offenbar war man in Deutschland weder an der Debatte um Fiktion oder Nichtfiktion noch an der Prosaform an sich interessiert. Das Projekt, den Prosa-Lancelot ins Deutsche zu übersetzen, wurde abgebrochen (vgl. Reil 1996). Man hat also in Deutschland auch im Bereich des Romans sehr genau gewusst (und wohl auch überlegt), welche Form man verwendete.

6 | Auch Zotz (vgl. 2005: 240f.) weist darauf hin, dass sogar bei den erwiesenen deutschen Kontrafakta romanischer Lieder die Idee der Einheit der Strophe nicht aufgegeben wird.

7 | Vgl. z.B. die Kaiserchronik, die in die 40er Jahre des 12. Jahrhunderts zurückreicht. 
Aber auch die altfranzösische Heldenepik wurde bei ihrer Übernahme in Deutschland in Paarreimverse umgesetzt. Die chanson de geste wurde bekanntermaßen in Laissen gedichtet, also in unregelmäßig langen Gruppen assonanter Langverse. Was diese Dichtungsform hergibt, hat im Grunde schon die Chanson de Roland gezeigt: längere, dichtere Laissen neben kurzen, schnell aufeinanderfolgenden; daneben variierende Wiederholungen (sogenannte laisses similaires) und unterschiedliche Perspektivierungen (sogenannte laisses parallèles). Diese sehr lockere und flexible Form hält sich in Frankreich über das ganze hohe Mittelalter, allenfalls werden die freien Verse zu regulären Alexandrinern; erst im 14. Jahrhundert erscheinen in Frankreich mise-en-prose von Heldenepen, die einen formalen Wechsel bringen. Das deutsche Rolandslied setzt den französischen Text jedoch in Paarreimverse um, und dasselbe tut Wolfram von Eschenbach, der fast ein halbes Jahrhundert später in seinem Willehalm das zweite französische Heldenepos bearbeitet. Alle späteren deutschen Chanson-de-gesteBearbeitungen folgen diesen beiden Vorbildern. Man hat also die französische Heldendichtung in Deutschland formal dem höfischen Roman angeglichen und ihr die Partikularität und Charakteristik der Laissen-Form abgenommen.

Parallel dazu entsteht in Deutschland eine Heldenepik eigener Art (vgl. Heinzle 1999; Millet 2008; Lienert 2015). Als literarische Gattung ist die deutsche Heldendichtung nicht sonderlich kohärent, weil die Werke, die wir unter diesem Namen gruppieren, stofflich, thematisch und formal sehr unterschiedlich sind. Gewiss, einige von ihnen bilden zweifellos literarische Reihen, weil sie sich erkennbar und beschreibbar aufeinander beziehen oder von gemeinsamen Konventionen geprägt sind; doch insgesamt bleibt die Gattung recht disparat. Das erkennt man bereits an ihrer Form. Von den 17 uns vollständig oder fragmentarisch erhaltenen Werken, sind zwölf in Strophen gedichtet, fünf in Reimpaarversen (Klage, Dietrichs Flucht, Dietrich und Wenezlan, Biterolf, Laurin). Zudem gibt es kurze und lange Reimpaarverse; und die Frage, ob der sogenannte Hildebrandston eine vierzeilige Strophe ist oder ob es sich nicht eher um paargereimte Langverse handelt, ist m.E. nicht hinlänglich untersucht worden. Doch auch wenn wir ihn als Strophe behandeln, ist das Bild der strophisch gedichteten Werke alles andere als einheitlich: Vier von ihnen stehen im sogenannten Bernerton (Goldemar, Eckenlied, Sigenot, Virginal), zwei im Hildebrandston (Ortnit und Wolf Dietrich, Rosengarten), eins in der Heunenweise (Wunderer D) und die übrigen fünf (Nibelungenlied, Kudrun, Rabenschlacht, Alpharts Tod, Walther) haben je eigene, sonst nicht weiter bekannte Strophenformen. Und mindestens zwei Werke (Laurin und Wunderer) sind sowohl in Reimpaarversen wie in Strophen überliefert. Es gibt also in Deutschland alles andere als eine einheitliche heldenepische Form. ${ }^{8}$

8 | Wir wissen zu wenig darüber, ob und wie diese Strophen gesungen wurden (vgl. Brunner 1970; 1979). Gewiss konnten sie gesungen werden, aber nichts weist darauf hin, dass sie immer gesungen werden mussten. In diesem Sinne könnten der Bernerton und die Titurelstrophe (vgl. Brackert/Fuchs-Jolie 2002: 25-40) auch verstanden werden als deutsche Vorboten der Lesestrophen, die sich etwas später im romanischen 
Aber man kann sagen, dass der strophische Erzählvers, in welcher Form auch immer, einen bedeutenden Anteil der deutschsprachigen Heldendichtung charakterisiert und damit einen nicht unerheblichen Anteil der Narrativik insgesamt. Bedenkt man, dass auch Wolframs Titurel und später dann vor allem Albrechts weit verbreiteter Jüngerer Titurel in Strophen gedichtet sind (was viel später dann in Füetrers Buch der Abenteuer gipfelt), dann erweitert sich dieses Spektrum, und die Alterität zur französischen Literatur wird sichtbar. Während es im 13. Jahrhundert in den erzählenden Großtexten der französischen Literatur praktisch nur die Prosa und die Laisse gibt (Versromane sind Ausnahmen), steht dem eine deutsche Erzählliteratur gegenüber, die nicht nur am Paarreimvers festhält, sondern die sogar eine gewisse Neigung zum strophischen Erzählen an den Tag legt.

Ich halte es nicht für wahrscheinlich, dass die Entscheidungen und Entwicklungen über die formalen Möglichkeiten der deutschen Literatur ohne einen Blick auf die französische vollständig verstanden werden können. Ich will nicht behaupten, dass z.B. das Entstehen strophischen Erzählens in Deutschland prinzipiell in ausdrücklicher Opposition zur Entstehung der Prosa in Frankreich stehe, denn dass das Nibelungenlied in Strophen gedichtet wurde, basiert zweifellos eher auf internen Entwicklungen wie z.B. ein Anschluss an mündliche Traditionen heroischen Singens. Aber ich kann mir bei Wolfram von Eschenbach, der mit Sicherheit einiges über die Bearbeitungen des Gralsstoffes in Frankreich wusste und damit wahrscheinlich auch von den neu aufkommenden Gral-Prosaromanen wie dem Perlesvaux gehört hatte und der sich gleichzeitig, spätestens bei der Arbeit am Willehalm, der Herausforderung des Nibelungenliedes stellen musste, durchaus vorstellen, dass er die Entscheidung, den Titurel in Strophen zu dichten, nicht allein, aber auch mit Blick auf Frankreich traf. Oder auf die Heldenepik bezogen: Sicherlich haben nicht alle strophischen Heldendichtungen des 13. Jahrhunderts die Form im Bewusstsein gewählt, etwas in Opposition zur französischen chanson de geste zu dichten. Doch ich kann mir kaum vorstellen, dass das deutsche Literaturpublikum, das zur Entwicklung und Verbreitung des Bernertons beigetragen hat, nicht genau gesehen hätte, dass mit dieser Form etwas geschaffen wurde, was anders war als in der französischen Literatur und damit neu und eigen und interessant. Dass in der deutschen Literatur also für längere Erzählungen die Prosa erst im 15. Jahrhundert aufkommt und dafür das 13. Jahrhundert geprägt ist vom Paarreimvers auf der einen Seite, der in Frankreich nur noch marginal ist, und von strophischen Erzählformen auf der anderen Seite (die in Frankreich inexistent sind), beruht gewiss auf internen Entwicklungen und Vorlieben, ist aber nur mit diesem Seitenblick auf die Romania in seinem vollen Ausmaß zu verstehen. Offenbar wollte man nicht immer nur im französischen Stil schreiben, sondern war auch selbstbewusst und ehrgeizig genug, neue Formen auszuprobieren.

Raum entwickelten. Damit soll jedoch ihre Nähe auch zur Liedlyrik keineswegs unterschlagen werden. 


\section{LITERATUR}

Asperti, Stefano (2002): La tradizione occitanica. In: Piero Boitani/Mario Mancini/ Alberto Varvaro (Hg.): Lo spazio letterario del medioevo. 2. II medioevo volgare. Rom, S. 521-554.

Bein, Thomas ( $\left.{ }^{15} 2013\right)$ : Walther von der Vogelweide. Leich, Lieder, Sangsprüche. Berlin. Billy, Dominique (1989): L’Architecture lyrique médiévale. Analyse métrique et modélisation des structures interstrophiques. Paris.

Brackert, Helmut/Fuchs-Jolie, Stephan (Hg.; 2002): Wolfram von Eschenbach. Titurel. Berlin/ New York.

Brunner, Horst (1970): Epenmelodien. In: Otmar Werner/Bernd Nauman (Hg.): Formen mittelalterlicher Literatur. Siegfried Beyschlag zu seinem 65. Geburtstag. Göppingen, S. 149-178.

Ders. (1979): Strukturprobleme der Epenmelodien. In: Egon Kühebacher (Hg.): Deutsche Heldenepik in Tirol. König Laurin und Dietrich von Bern in der Dichtung des Mittelalters. Bozen, S. 300-328.

Bumke, Joachim (1986): Höfische Kultur. Literatur und Gesellschaft im hohen Mittelalter. München.

Cirlot, Victoria (1987): La novela artúrica. Orígenes de la ficción en la cultura europea. Barcelona.

Frank, István (21966): Répertoire métrique de la poésie des troubadours. Paris.

Di Girolamo, Costanzo (1979): Elementi di versificazione provenzale. Neapel.

Ders. (1989): I trovatori. Turin.

Hasenohr, Geneviève (1990): Les recueils lyriques. In: Jean Martin/Jean Vezin (Hg.)

Mise en page et mise en texte du livre manuscrit. Paris, S. 329-334.

Heinzle, Joachim (1999): Einführung in die mittelhochdeutsche Dietrichepik. Berlin.

Herchert, Gaby (2010): Einführung in den Minnesang. Darmstadt.

Lienert, Elisabeth (2015): Mittelhochdeutsche Heldenepik. Eine Einführung. Berlin.

Meneghetti, Maria Luisa (1984): Il pubblico dei trovatori. Ricezione e riuso dei testi lirici cortesi fino al XIV secolo. Modena.

Millet, Victor (2008): Germanische Heldendichtung im Mittelalter. Eine Einführung. Berlin.

Mölk, Ulrich (1982): Trobador-Lyrik. München / Zürich.

Moser, Hugo/Tervooren, Helmut (381998): Des Minnesangs Frühling. Erneut revidierte Aufl. Stuttgart.

Müller, Ulrich/Bennewitz, Ingrid/Spechtler, Franz Viktor/Weichselbaumer, Ruth (Hg.; 2007): Neidhart-Lieder. Texte und Melodien sämtlicher Handschriften und Drucke. Berlin.

Pillet, Alfred/ Carstens, Henry (1933): Bibliographie der Troubadours. Halle.

Räkel, Hans-Herbert S. (1986): Der deutsche Minnesang. Eine Einführung mit Texten und Materialien. München.

Reil, Cornelia (1996): Liebe und Herrschaft. Studien zum altfranzösischen und mittelhochdeutschen Prosa-Lancelot. Tübingen.

Rieger, Dietmar (1983): Die altprovenzalische Lyrik. In: Heinz Bergner (Hg.): Lyrik des Mittelalters. Probleme und Interpretationen. Bd. 2. Stuttgart, S. 197-390. 
Riquer, Martín de (1983): Los trovadores. Historia literaria y textos. Barcelona.

Schweikle, Günther (21995): Minnesang. Stuttgart.

Tervooren, Helmut (1995): Sangspruchdichtung. Stuttgart.

Zotz, Nicola (2005): Intégration courtoise. Zur Rezeption okzitanischer und französischer Lyrik im klassischen deutschen Minnesang. Heidelberg. 
